\title{
Association between TNF-a secretion levels in major depressive disorder and symptoms' severity, before and after mitogen stimulation
}

\author{
Platon Christopoulos*1, Kokona Hajantoni ${ }^{1}$, Anna Lisa Delastik1, \\ Athanasia Mouzaki ${ }^{1}$ and Philippos Gourzis ${ }^{2}$
}

\author{
Address: ${ }^{1}$ Division of Haematology, Dpt. of Internal Medicine, University Hospital of Patras, Patras Greece and ${ }^{2}$ Department of Psychiatry, \\ University Hospital of Patras, Patras, Greece \\ * Corresponding author
}

\author{
from International Society on Brain and Behaviour: 3rd International Congress on Brain and Behaviour \\ Thessaloniki, Greece. 28 November - 2 December 2007 \\ Published: 17 April 2008 \\ Annals of General Psychiatry 2008, 7(Suppl I):S243 doi:I0.I I86/I744-859X-7-SI-S243
}

This abstract is available from: http://www.annals-general-psychiatry.com/content/7/SI/S243

(c) 2008 Christopoulos et al.; licensee BioMed Central Ltd.

\section{Background}

It is well established that cytokines signal the brain and serve as mediators between immune and nerve cells. Recent research findings have led to speculation that the neuroimmunologic consequences of cytokine levels might be involved in the pathogenesis of depression. Although TNF-a is required for normal immune responses, its overexpression has severe pathological consequences. The present study was undertaken to determine whether TNF-a secretion levels before and after mitogenic stimulation differ between depressed patients and matched healthy controls during clinical improvement.

\section{Materials and methods}

Nineteen subjects ( 8 males, 11 females, mean age 63.2 years, range 48-71), inpatients and outpatients of the Department of Psychiatry who met DSM-IV criteria for a principal diagnosis of Major Depressive Disorder (MDD) were enrolled. The control group consisted of twenty, ageand sex-matched healthy blood donors (mean age 59.12 years, S.D, $+/-21$ ). The severity of depressive symptoms was rated using the Hamilton Depression Scale (HAM-D, 17 Item Score). Peripheral Blood Mononuclear cells were isolated and cultured at a concentration of 106 cells $/ \mathrm{ml}$ for 72 hours in plain culture medium (RPMI containing $10 \%$ Fetal Calf Serum and 1\% Penicillin/Streptomycin) and in the presence of PMA and Ionomycine. Cytokine concentrations were determined in the supernatants by enzyme-linked immunosorbent assay (ELISA). Strict exclusion criteria concerning factors that might have influenced immune function and cytokine secretion levels were applied.

\section{Results}

TNF-a secretion levels were significantly higher in patients suffering from Major Depressive Disorder compared to healthy controls. Higher HAM-D Score was often combined with increased production of the cytokine but not always in a linear way. Mitogen stimulated TNF-a secretion also seems to be affected by symptoms severity and recovery.

\section{Conclusions}

Our data indicate a significant positive association between Depression and increased TNF-a secretion levels. However we failed to demonstrate a linear association between symptoms severity and TNF-a secretion. Altogether these data suggest an immune system activation in patients with MDD, although the relationship of these immune anomalies to the pathophysiology of MDD still remains far from clear. However, should this hypothesis be confirmed, the implications are far-reaching and include new ways of both treating and preventing major depression. 